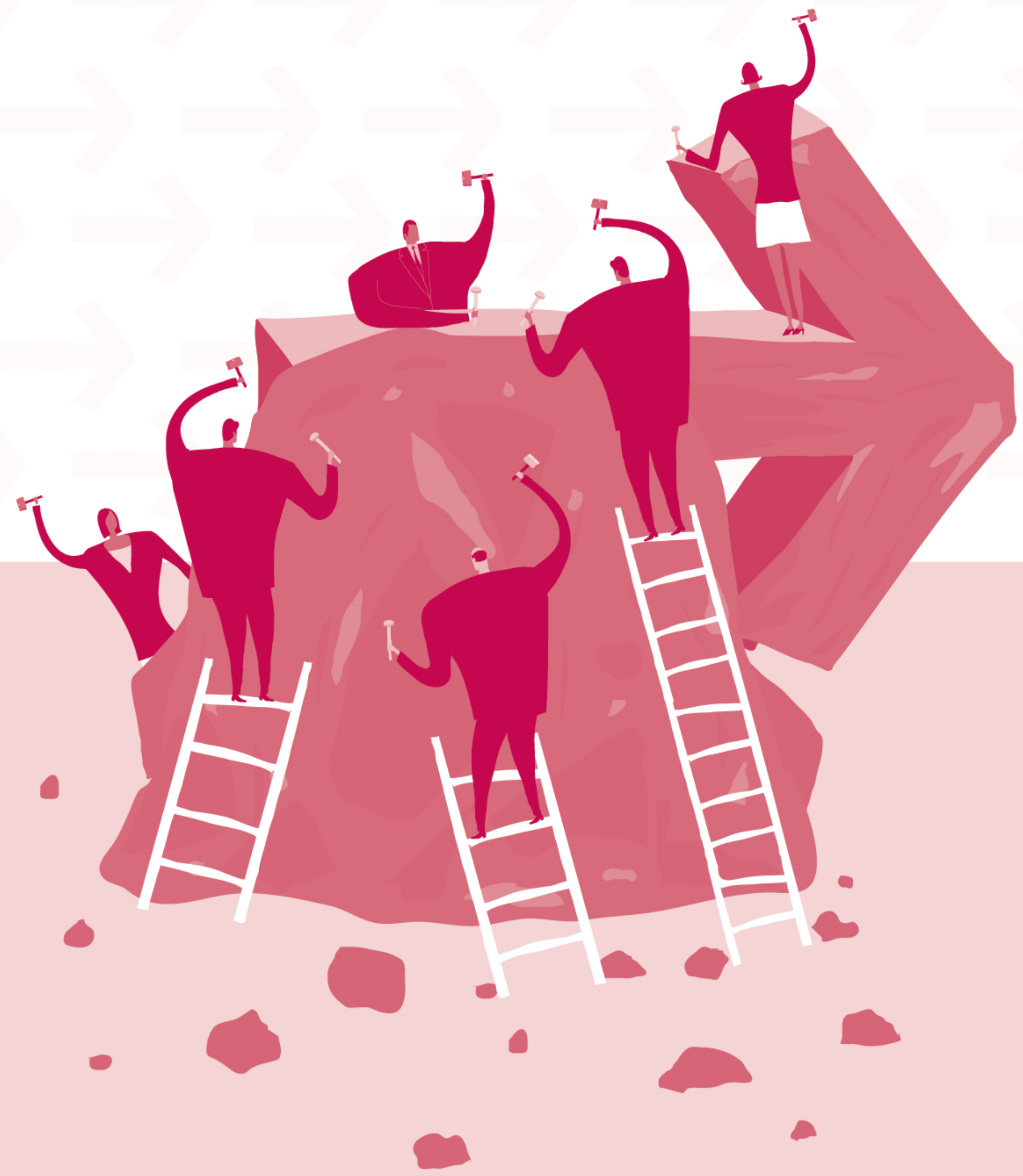




\section{Orientação para o cliente, Comunicação e Cultura Organizacional}

Roberto Coda

- Livre-docente, doutor e mestre em Administração de Empresas pela Faculdade de Economia, Administração e Contabilidade da Universidade de São Paulo (FEA-USP)

- Professor associado do Departamento de Administração da FEA-USP

- Professor e pesquisador do Centro Universitário Fecap (Fundação Escola de Comércio Álvares Penteado) junto ao Programa de Mestrado em Ciências Contábeis

-rcoda@fecap.br

Ana Carolina Maciel da Silva

- Mestre em Administração de Empresas pela Fecap

- Pós-graduada em Administração de Empresas pela Fundação Getúlio Vargas (FGV)

- Bacharel em Ciência da Computação pela Universidade Federal de Uberlândia (UFU)

- anacarolinamaciel@hotmail.com

Mauro Neves Garcia

- Doutor e mestre e em Administração de Empresas pela FGV-SP

- Professor e pesquisador da Universidade Municipal de São Caetano do Sul (Imes) junto ao Programa de Mestrado em Administração

•mnevesg@gmail.com

Dirceu da Silva

- Doutor em Educação pela Faculdade de Educação da USP

- Mestre e bacharel em Física pelo Instituto de Física da USP

- Especialista em Métodos Quantitativos

- Professor e pesquisador do Programa de Pós-Graduação da Imes

- Professor da Faculdade de Educação da Unicamp

-dirceu@imes.edu.br 
Resumo

A orientação ao cliente é princípio essencial do conceito de Marketing desde sua criação nos anos 1950. Hoje, é necessário que todos os empregados incorporem essa crença em seus comportamentos, pois percebem que agir assim é explicitamente comunicado pela própria cultura organizacional. Este trabalho utiliza análise fatorial em pesquisa com 250 sujeitos para identificar construtos culturais que, quando presentes em uma organização, efetivamente apóiam uma orientação ao cliente, promovendo um diálogo entre Marketing, Gestão de Pessoas e Comunicação.

PALAVRAS-CHAVE: CULTURA ORGANIZACIONAL • MOTIVAÇÃO • ORIENTAÇÃO PARA O CLIENTE - COMUNICAÇÃo

\section{Abstract}

Client orientation has been a major Marketing principle since its creation in the 1950 s. Presently it is necessary that all employees be committed to this belief, merging it into their professional behavior, to the extent that they perceive that acting in this way is explicitly communicated by the organizational culture in itself. This paper applies factor analysis to a survey with 250 respondents aimed at identifying cultural concepts associated with client orientation, promoting a dialogue between Marketing, People Management and Communication.

KEYWORDS: ORGANIZATIONAL CULTURE • MOTIVATION • CLIENT ORIENTATION • COMMUNICATION

\section{Resumen}

La orientación al cliente es un principio esencial del concepto de Marketing desde su creación, en la década de 1950. Hoy es necesario que todos los empleados incorporen esta creencia a sus comportamientos, pues se percibe que actuar así es comunicado explícitamente por la propia cultura de la organización. Se utiliza el análisis factorial aplicado a una investigación con 250 personas para identificar construcciones culturales que, cuando se encuentran en una organización, efectivamente dan apoyo a una orientación al cliente y promueven un diálogo entre el Marketing, la Gestión de Personal y la Comunicación.

PALABRAS CLAVE: CULTURA ORGANIZACIONAL - MOTIVACIÓN • ORIENTACIÓN AL CLIENTE • COMUNICACIÓN 
orientação ao cliente é o princípio fundamental do conceito de Marketing cria-
do nos anos 1950. Drucker (1954), um dos principais divulgadores do concei-
to naquela época, argumentava que o propósito de uma organização era criar um cliente satisfeito, sendo que, dentre todos os stakeholders, o cliente deveria estar em primeiro lugar. Nessa linha, o autor ainda propunha que Marketing não era uma atividade específica, mas, sim, uma filosofia para o negócio como um todo, a ser dirigido segundo a ótica do cliente e suas definições de valor.

O consumidor atual julga os produtos e serviços com base no seu valor fundamental, definido pela relação entre custo e benefício. O próprio conceito de Marketing precisou ser repensado e, no início dos anos 1990, foi redefinido para contemplar a geração e a entrega de valor ao cliente, como forma de obter vantagem competitiva (KOTLER, 2000; WEBSTER, 1994b). Assim, o novo conceito de Marketing é definido em torno do conceito de cadeia de valor (PORTER, 1991), que incorpora o foco tanto no cliente - inerente ao conceito de Marketing - como na capacidade, recursos e habilidades da empresa - inerente ao conceito de estratégia.

A literatura sugere, ainda, que desenvolver uma cultura organizacional apropriada está entre as capacidades, recursos e fatores necessários à implementação do conceito de Marketing em uma organização. Em 1989, Deshpande \& Webster publicaram um artigo que representa um marco para os estudiosos de Marketing, trazendo à tona a importância de se encorajar o desenvolvimento de uma corrente de pesquisa sobre Cultura Organizacional em Marketing. Narver e Slater (1998) também defendem que a orientação ao cliente deve estar associada a um sistema de crenças e valores apropriado, caso contrário, qualquer que fosse a cultura organizacional, uma orientação ao cliente poderia ser facilmente implementada a qualquer tempo, não sendo, porém, o que se observa.

A Cultura Organizacional refere-se a um sistema de significados partilhados e mantidos por seus membros que identifica uma organização, distinguindo-a de outras organizações (ROBBINS, 1999). Embora não exista uma cultura organizacional certa ou errada, boa ou má, o que precisa existir é um alinhamento entre pessoas, cultura e objetivos da organização para que seja possível realizar estratégias, inclusive as de mercado, caso contrário, a cultura organizacional pode ser uma barreira ao processo e não uma aliada. Existem, portanto, valores e crenças culturais que favorecem a orientação ao cliente e que devem ser buscados e cultivados nas empresas que valorizam essa orientação. 
ORIENTAÇÃO PARA O CLIENTE, COMUNICAÇÃO E CULTURA ORGANIZACIONAL •

ROBERTO CODA, ANA CAROLINA MACIEL DA SILVA, MAURO NEVES GARCIA E DIRCEU DA SILVA

Com base em uma revisão da literatura sobre orientação ao cliente e Cultura Organizacional e em teste empírico de um conjunto mais amplo de indicadores realizado junto a organizações brasileiras, o presente estudo tem como objetivo geral desenvolver um instrumento de medição válido para os construtos valores culturais e orientação ao cliente, por meio da investigação das variáveis e fatores que possam formar esses conceitos. Representa, portanto, uma tentativa de identificar valores ou traços culturais que favoreçam ou contribuam para que uma organização demonstre uma efetiva orientação ao cliente externo, a fim de que esses valores possam ser comunicados eficazmente aos clientes internos da organização.

\section{Cultura organizacional e orientação ao cliente}

O surgimento do conceito de Marketing vem em resposta a mudanças profundas que as organizações enfrentavam em um contexto pós-Segunda Guerra Mundial e para o qual o pensamento de Marketing vigente já não era mais apropriado. A responsabilidade do Marketing, até então, era estimular a demanda pelos produtos, persuadir o cliente a comprar o que a empresa produzia e realizar uma venda. O foco, portanto, era em produtos ou na capacidade produtiva, e não em consumidores, seus desejos e necessidades. $\mathrm{O}$ aumento da competição na maioria dos mercados e do poder de compra dos consumidores exige uma verdadeira orientação para o cliente como estratégia de negócios. Assim, os anos 1960 e o início de 1970 são caracterizados como a época de ouro do Marketing.

Entretanto, no decorrer dos anos 1970, o compromisso com o cliente se desvaneceu e o planejamento estratégico, carregado de imperativos financeiros, passou a ganhar preferência entre os administradores. As organizações desviaram a sua atenção da satisfação do cliente como fonte de vantagem competitiva e lucratividade no longo prazo. Webster (1994a) argumenta que tal fato ocorreu porque o conceito de Marketing, proposto por Drucker, deixava algumas lacunas em sua definição. Entre elas, destaca a falta de um componente mais tático que apontasse aos administradores como efetivamente uma empresa poderia ser orientada ao cliente, que tipo de atividades ou comportamentos eram necessários. A orientação ao cliente demonstrava ser um conceito difícil de operacionalizar, observar, medir e comunicar, além do que muitas organizações não acreditavam que a orientação ao cliente pudesse garantir sua sobrevivência e desempenho financeiro.

Nos anos 1980, muitas empresas, principalmente americanas, experimentaram as conseqüências de se distanciar do cliente. O mercado americano foi invadido por concorrentes estrangeiros que, rapidamente, conquistaram participação, oferecendo aos clientes produtos adequados às suas necessidades.

A partir dos anos 1990, alguns acontecimentos modificaram o cenário competitivo em 
muitos países. Presenciou-se a desregulamentação de mercado, trazendo a livre concorrência em setores econômicos antes totalmente fechados. A privatização também trouxe novas configurações a esse cenário em que empresas públicas, antes protegidas em seus monopólios, viram-se inseridas em um ambiente agora de competição. No Brasil, por exemplo, destacam-se as privatizações nos setores de telecomunicações e elétrico. E ainda, a competição global tornou-se um fato da vida econômica de países industrializados, bem como das economias em desenvolvimento.

Em face dessa realidade, determinados autores (KOTLER, 2000; WEBSTER, 1994b) propõem a ampliação do conceito de Marketing definido por Drucker nos anos 1950. Esses autores argumentam que o velho conceito de Marketing nasceu em um contexto pós-guerra marcado por economias escassas, demanda reprimida por parte dos consumidores e em que produtos eram comprados mais com base no conceito que o produto transmitia e no estilo de vida do consumidor do que nos benefícios específicos de performance que o produto entregava. Portanto, o novo conceito de Marketing, compartilhado também por Slater e Narver (1994), contempla o processo de definir, desenvolver e entregar valor ao cliente, sendo que uma empresa orientada ao cliente continuamente examina as fontes alternativas de vantagem competitiva sustentável, a fim de saber como ela pode ser mais efetiva em criar valor superior igualmente sustentável para seus clientes atuais e futuros. Em suma, o novo conceito de Marketing propõe que o negócio seja definido de fora para dentro, sendo imperativo estar bem informado sobre o cliente e deixar que ele defina valor.

Em 1990, Kohli e Jaworski publicaram um artigo com o objetivo de delinear o domínio do construto orientação ao mercado, prover uma definição operacional, desenvolver um inventário de proposições e construir um modelo compreensível para direcionar futuras pesquisas. Enfatizaram a criação de um modelo teórico para o construto. Para eles, uma empresa orientada ao mercado é aquela que implementa o conceito de Marketing e por isso define orientação ao mercado de modo a facilitar a operacionalização desse conceito, ou seja,

"orientação ao mercado é a geração de inteligência de mercado sobre as necessidades atuais e futuras dos clientes em toda a organização, a disseminação da inteligência para todos os departamentos e a resposta da organização frente às informações obtidas". (KOHLI; JAWORSKI, 1990, p. 6)

Por outro lado, uma empresa é orientada ao mercado quando sua cultura está sistemática e totalmente comprometida com a contínua criação de valor superior ao clien- 
ORIENTAÇÃO PARA O CLIENTE, COMUNICAÇÃO E CULTURA ORGANIZACIONAL •

ROBERTO CODA, ANA CAROLINA MACIEL DA SILVA, MAURO NEVES GARCIA E DIRCEU DA SILVA

te. Isso significa coletar e coordenar informações sobre os clientes, competidores e outros significativos influenciadores de mercado, como fornecedores e reguladores, para usar na construção de valor (SLATER; NARVER, 1994).

Colocar o cliente em primeiro lugar pode representar uma quebra de paradigma para muitas empresas e um desafio interno, na mente das pessoas e em suas atitudes, desde o alto executivo até níveis mais baixos da estrutura organizacional. Conforme Whiteley (1999), as empresas que colocam o cliente em primeiro lugar têm como principais características: pensar e falar muito em seus clientes; avaliar as percepções de seus clientes rotineiramente; tender a resolver questões de prioridade, considerando o impacto mais provável sobre o cliente como critério; ceder, fazer concessões ou acrescentar valor para o cliente em situações, como já colocado, que demandam a oferta de serviços nãorotineiros, adotando a política de fazer o que é necessário; recuperar-se conscienciosamente de erros e se penitenciar com o cliente que recebeu um tratamento inadequado.

Kotler (2000) observa que empresas que implementam a inteligência de mercado estão melhorando seu conhecimento do cliente, convidam os clientes a participarem do projeto do produto, estão prontas para tornar seus produtos, serviços e condições mais flexíveis. Em suma, encontraram maneiras de fornecer um valor superior a seus clientes. Também, nelas o pensamento e as práticas inovadoras são estimulados e estão associados a maiores níveis de orientação ao cliente.

Webster (1994b) propõe que a orientação ao cliente requer atenção em três níveis: estratégia, estrutura e cultura. Para esse autor, a estratégia deve estar focada em criar satisfação do cliente por meio da entrega de valor superior. A estrutura organizacional deve ser desenhada para implementar a estratégia e nela a função de Marketing deve estar pulverizada em todos os departamentos, ou seja, satisfazer o cliente é responsabilidade de cada indivíduo na empresa. A cultura deve nutrir valores e crenças que coloquem o cliente em primeiro lugar em todas as atividades da empresa.

Day (1998) também incorpora a Cultura Organizacional em seu modelo teórico de orientação a clientes e mercado, definida como a habilidade superior de compreender, atrair e reter clientes valorosos. Enfatiza que tudo emana da cultura certa - aquela que está dedicada a entregar valor superior ao cliente. A cultura reforça-se pelo processo de pensamento estratégico orientado para o exterior e impulsiona a habilidade de estabelecer relações próximas com os clientes valorosos. E, finalmente, para ser efetiva, a cultura ainda deve ser reforçada pela estrutura organizacional correta para definir o elo entre atividades, incentivos e sistemas de informação para manter toda a empresa em uma direção única.

O termo Cultura tem suas origens na antropologia e é também definido por Tylor (1871), citado por Cabrera e Bonache (1999, p. 53), como "um todo complexo que inclui conhecimento, crenças, artes, moral, leis, costumes e outras capacidades e hábi- 
ORIENTAÇÃO PARA O CLIENTE, COMUNICAÇÃO E CULTURA ORGANIZACIONAL •

ROBERTO CODA, ANA CAROLINA MACIEL DA SILVA, MAURO NEVES GARCIA E DIRCEU DA SILVA

tos adquiridos pelo homem como membro de uma sociedade”. Quando se estuda o mesmo fenômeno no contexto das organizações, a Cultura é conhecida como Cultura Corporativa ou Cultura Organizacional, guardando as mesmas complexidades e amplitudes do seu termo original.

Pode-se dizer que o estudo da Cultura Organizacional é recente, sendo pioneiramente introduzido pelo autor Pettigrew, em 1979, na literatura organizacional. E, por ter cerca de 30 anos de estudo, ainda não há definição única para Cultura Organizacional, o que aponta para a necessidade de um maior amadurecimento e fortalecimento do tema em trabalhos acadêmicos. Como coloca Hofstede et al. (1990), apesar de não existir um consenso sobre sua definição, algumas características da Cultura Organizacional certamente são compartilhadas entre os estudiosos: (1) é holística, permeia toda a organização; (2) é historicamente determinada; (3) relaciona-se a conceitos originados na antropologia; (4) é socialmente construída; (5) é intangível; (6) é difícil de mudar; e (7) para ser compartilhada, precisa ser comunicada aos integrantes da organização.

Thompson e Luthans (1994) afirmam que, apesar de não haver uma concordância do que é cultura e quão arraigada ela é na organização, existe um consenso geral de que ela é um componente importante que afeta a performance e o comportamento dos integrantes de uma organização. As interações e a comunicação entre as pessoas da organização são uma manifestação da cultura e, para esses autores, a cultura somente pode ser mudada por meio de mudanças nos comportamentos das pessoas.

Na mesma linha, Dessler (1998) afirma que valores e normas que os empregados compartilham - a cultura organizacional - são crenças básicas sobre o que se deve ou não fazer, e o que é importante ou não. Elas guiam e canalizam o comportamento das pessoas. Desse modo, para o autor, conduzir e influenciar as pessoas depende, em parte, de influenciar essas normas e valores usados como referências comportamentais.

Analisando diferentes autores, Schneider (1990) afirma haver duas correntes de estudos sobre Cultura Organizacional: uma corrente analisa e investiga a cultura como algo que a organização tem, e a outra, como algo que a organização é. Além disso, por ser um campo de estudo muito amplo, o que tornaria o estudo de todos os elementos culturais praticamente uma tarefa impossível, muitos autores se dedicam a aprofundar suas investigações em determinados elementos culturais, que variam de acordo com o modelo por eles proposto.

Estudiosos que fazem parte da primeira corrente (HOFSTEDE, 1991; ROUSSEAU, 1990; SCHEIN, 1997) desenvolveram modelos ou conceitos que investigam a cultura como característica ou traço da organização. E, nessa linha, o modelo integrado de cultura corporativa proposto por Dessler (1998) representa, de certa forma, uma consolidação dos modelos propostos pelos estudiosos da primeira corrente. 
Partindo do centro para a periferia (Figura 1), o modelo propõe que a cultura de uma organização emerge de pressupostos básicos, valores e crenças, padrões de comportamento e de suas manifestações mais visíveis e diretas, como os artefatos (linguagem, mitos, arquitetura etc.).

Figura 1. Modelo de James Hunt (1998)

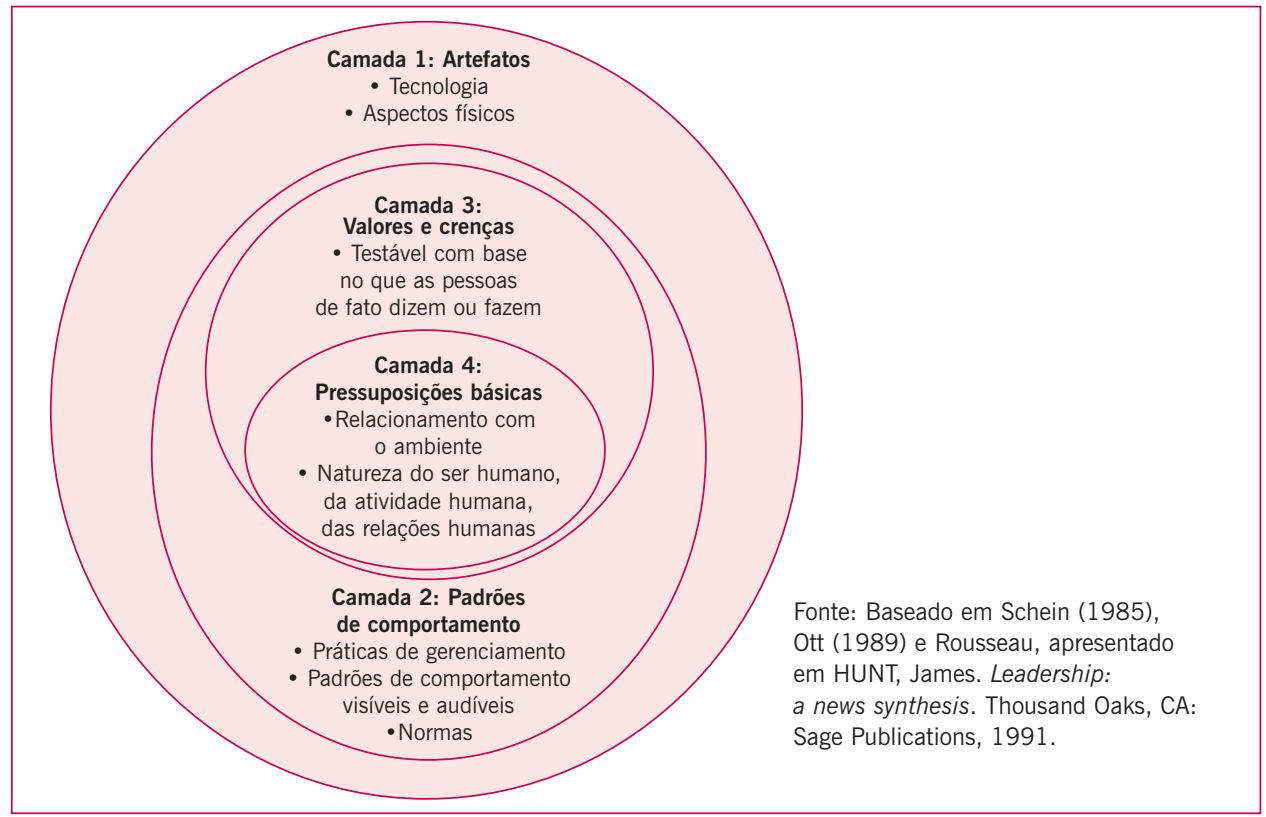

Fonte: Dessler (1998)

Hunt (1991), citado por Dessler (1998), propõe a existência de uma cadeia de influências: pressuposições influenciam valores, que, por sua vez, influenciam o comportamento e as ações, que definem os artefatos físicos, como, por exemplo, as regras escritas ou formais. Segundo o modelo, valores e crenças (como "O cliente está sempre certo" ou "Não seja burocrático") são padrões de referência que indicam o que se deve ser, e não o que se é efetivamente. Dessler (1998) afirma que valores e crenças declarados muitas vezes se diferem do que de fato as pessoas valorizam e acreditam. Se isso acontece, o comportamento das pessoas é o sinal que revela esse desalinhamento. Por essa razão, é possível inferir os valores compartilhados com base nas atitudes das pessoas.

Robbins (1999) afirma que a cultura organizacional tem várias funções dentro de uma empresa: constituir papel de definição de fronteira, ou seja, criar distinções entre uma organização e outras; transmitir um sentido de identidade para os membros da organização; facilitar a geração de comprometimento a alguma coisa maior do que o auto-in- 
ORIENTAÇÃO PARA O CLIENTE, COMUNICAÇÃO E CULTURA ORGANIZACIONAL •

ROBERTO CODA, ANA CAROLINA MACIEL DA SILVA, MAURO NEVES GARCIA E DIRCEU DA SILVA

teresse. Uma cultura é forte quando os valores centrais da organização são intensamente mantidos e amplamente partilhados; por essa razão, tem grande influência no comportamento dos seus membros. Do ponto de vista do empregado, a cultura é valiosa porque reduz a ambigüidade, comunicando aos empregados como as coisas devem ser feitas e o que é importante fazer. Mais do que isso, a cultura organizacional é o elo que une os líderes na efetiva implementação das estratégias organizacionais e a ausência desse elo pode provocar efeitos desastrosos à organização (OVERHOLT; GRANNEL, 2002).

\section{O papel da política de comunicação nas organizações}

Desde a década de 1990, a Comunicação Organizacional como tema de pesquisa vem recebendo atenção prioritariamente como um instrumento voltado para a reunião e transmissão de informações que facilitem a consecução dos objetivos organizacionais.

A partir do início dos anos 2000, a política de comunicação começou a ser vista como um facilitador para a obtenção do sucesso organizacional, principalmente se exercer o papel de auxiliar os empregados a compreender a natureza do negócio, os valores e a cultura da organização, alinhando, assim, os objetivos organizacionais com os individuais. Na tentativa de explicitar quais as características da política de comunicação interna que efetivamente contribuem para essas finalidades, Sallie-Dosunmu (2001) enfatiza cinco diferentes aspectos essenciais - precisa, clara, amigável, positiva e proativa.

Outro aspecto relevante que pode ser encontrado na literatura em relação ao papel da Comunicação Organizacional interna e externa é que ambas precisam ser concebidas de forma estratégica. E para serem consideradas estratégicas, necessitam promover o equilíbrio entre elementos-chave presentes na vida organizacional. Para garantir a eficácia da comunicação, os profissionais da área devem alinhar as informações com as questões internas da organização, tentando aprender mais sobre a dinâmica e o comportamento da organização, sobre o clima e a cultura organizacional e sobre a construção de confiança, unindo todos esses aspectos no que Potter (2003) denomina efetiva gestão estratégica da comunicação. Dessa forma, a política de comunicação somente assumiria esse caráter estratégico quando estivesse voltada para clarificar a cultura da organização, seja a atual, seja aquela que pretenda incorporar novos valores como, por exemplo, a preocupação com clientes internos e externos, principalmente ao transmitir de modo claro as regras e princípios que viabilizam o comportamento organizacional desejado.

Para especialistas em mudança organizacional, o papel da política de comunicação é auxiliar a transformar a estratégia em ação (AYELET, 2004). Esse ângulo é de especial interesse para o enfoque conferido ao presente artigo, uma vez que a orientação para o cliente representa uma estratégia para materializar o próprio conceito de Marketing dentro de uma organização. 
ORIENTAÇÃO PARA O CLIENTE, COMUNICAÇÃO E CULTURA ORGANIZACIONAL •

ROBERTO CODA, ANA CAROLINA MACIEL DA SILVA, MAURO NEVES GARCIA E DIRCEU DA SILVA

Um estudo de caso realizado durante 2004 em uma empresa do setor público australiano por Waterhouse e Lewis colabora para mapear a complexidade associada ao papel da Comunicação Organizacional. Os resultados identificados apontam para a existência de um contínuo que traduz o efetivo papel da comunicação na transmissão de objetivos privilegiados pela direção ou de mudanças culturais. Tal contínuo varia desde um excesso de informações até sua total ausência, ou mesmo a falta de consulta às pessoas envolvidas com as mudanças. A conclusão do trabalho enfatiza que a política de comunicação, como meio condutor de informações, tem que evoluir para o diálogo e se processar por meio dele, não sendo somente do tipo cúpula-base, mas também ocorrendo no sentido inverso, fato que viabilizará a possibilidade de aprendizado e de incorporação dos novos valores pretendidos.

Quando o foco se volta para o campo das Relações Públicas (RP), de especial interesse para o tema deste artigo é o trabalho contendo uma discussão teórica e prática sobre o papel desse setor nas organizações, chamando a atenção para a imagem negativa associada à área de RP. A pesquisa realizada por Cheney e Christensen (2006) deixa claro que a área de RP tende a negligenciar os assuntos internos da organização, tais como os valores essenciais da própria organização, ou seja, sua cultura, assim como opiniões e preferências dos empregados, fundamentando a perspectiva segundo a qual torna-se necessário colocar em prática amplas e persuasivas campanhas de relacionamento e também de comunicação que, espera-se, irão gerar os efeitos comportamentais esperados e positivos, bem como de uma forma ampla e cumulativa.

\section{Comunicação e cultura organizacional}

A maior parte dos trabalhos relativos à interface entre cultura organizacional e comunicação deixa clara a importância da última no sentido de efetuar a transmissão e a divulgação dos valores, missão, crenças e, em última análise, o credo empresarial para todos os integrantes de uma organização.

Um caso emblemático e ilustrativo sobre a eficácia da Comunicação Organizacional refere-se à mudança cultural levada a efeito nas lojas GAP, viabilizada por meio de ampla campanha comunicacional interna cujo objetivo foi o engajamento dos funcionários nos novos valores e crenças que a empresa passou a julgar conveniente adotar. Uma equipe de comunicação foi formada no início de 2004, a fim de planejar a divulgação do que foi denominado o novo DNA da GAP. A ação inicial previu uma estratégia cúpula-base junto aos 2.000 empregados que exerciam posição de liderança. Em seguida, foi definido um conjunto de informações voltado para manter vivos na mente dos demais 150.000 funcionários das lojas espalhadas pelo mundo os novos propósitos, valores e comportamentos (PVCs) para atender as novas diretrizes culturais. Os meios de comunicação utilizados variaram desde uma página especial na intranet até cartas enviadas diretamente aos líderes, além da publicação de um jornal interno pa- 
ra relato de histórias de sucesso e reconhecimento daqueles colaboradores efetivamente engajados na modelagem dos novos valores culturais. A avaliação final do projeto apresentou resultado positivo, uma vez que a maioria dos gerentes consultados declarou contar com as informações e instrumentos de comunicação necessários para apoiar a entronização dos novos valores culturais (NASH, 2005).

Em outro trabalho de pesquisa junto ao setor público americano voltado para a descoberta de características organizacionais desejáveis, Moyniham e Pandey (2006) novamente explicitam a interface entre comunicação e cultura organizacional. Uma das hipóteses levantadas foi a de que o uso de canais internos de comunicação facilitaria enfatizar determinados valores organizacionais como, por exemplo, foco nos resultados ou nos clientes. Das onze variáveis dependentes estudadas, a comunicação interna foi aquela que apresentou o maior poder explicativo da variável independente orientação para resultados, como valor cultural. A pesquisa revelou que era maior a orientação para resultados nas organizações em que as lideranças comunicavam tarefas, direção estratégica e forneciam feedback sobre o efetivo desempenho no trabalho. Além disso, os empregados declararam contar com canais específicos para comunicar à alta direção suas perspectivas sobre os problemas organizacionais e receber de seus pares efetivo suporte para o andamento dos trabalhos.

Falta de comunicação ou comunicação ineficiente são também apontadas como razões que tornam a implementação de mudanças culturais um processo complexo e nem sempre de sucesso. Um estudo comparativo sobre fatores facilitadores da mudança cultural em organizações, envolvendo empresas como a Alcoa, a British Telecom e a Crown House Engineering, realizado por Price e Chahal (2006), enfatiza, entre outros, a importância da Comunicação Organizacional. O êxito da mudança cultural ocorreu quando os programas de comunicação interna sobre esse processo estiveram baseados em informações mensais do tipo briefing a respeito do andamento e dos pressupostos da mudança, assim como na auditoria sobre a eficácia permanente dos canais de comunicação adotados. Problemas ocorreram quando a comunicação inicial, que deveria fluir da alta administração, foi lenta e inespecífica, levando ao desconforto dos empregados e à baixa produtividade.

A manutenção de linhas e canais de comunicação abertos é considerada igualmente como um aspecto facilitador da aquisição de características positivas da cultura de uma organização. Isso implica, segundo Edelman (2006), implementar uma política de portas abertas em todos os níveis da empresa, aspecto que fará o feedback fluir de modo

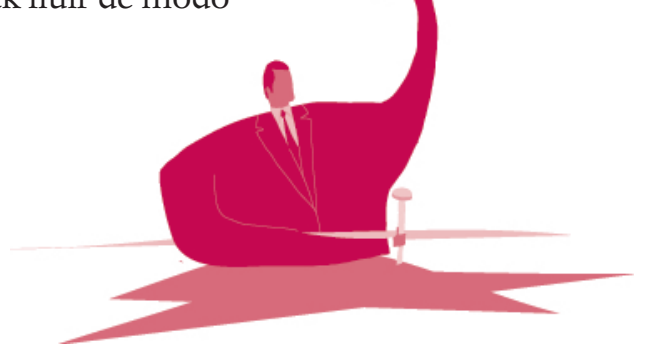


ORIENTAÇÃO PARA O CLIENTE, COMUNICAÇÃO E CULTURA ORGANIZACIONAL •

ROBERTO CODA, ANA CAROLINA MACIEL DA SILVA, MAURO NEVES GARCIA E DIRCEU DA SILVA

livre e sem restrições, fora da hierarquia organizacional formal. Embora o autor reconheça que a autoridade formal deva ser respeitada, contribuições de pessoas de todos os níveis precisam ser valorizadas e incentivadas, caracterizando um fluxo de comunicação ascendente, baseado no pressuposto de que são aqueles mais próximos do problema geralmente os que melhor sabem o que fazer para sua resolução.

Dentro de uma outra linha de investigação para explicitar o papel da Cultura Organizacional, ligando-a à teoria comunicacional denominada sensemaking, torna-se relevante para o contexto do presente artigo examinar os resultados obtidos por Ravasi e Schultz (2006) em pesquisa longitudinal realizada na Bang \& Olufsen, produtora dinamarquesa de sistemas de áudio e vídeo. Para esses pesquisadores, as ações de liderança deveriam estar alicerçadas em suas avaliações e reavaliações a respeito da identidade organizacional. Acrescentam também que a primeira etapa de um processo de mudança cultural necessita estar fundamentada em uma fase de busca de sentido associada a novas ou revistas concepções sobre aspectos centrais e distintos da organização, sentido esse que precisa ser transmitido de modo eficaz para que possa ser devidamente compartilhado.

Finalmente, a importância da variável comunicação é evidenciada por Landale (2007) com o objetivo de chamar a atenção para a necessidade de aquisição de novos valores para a cultura de uma organização. A pesquisa realizada trata da variável respeito e sensibilidade em relação às pessoas (inteligência emocional) como aspecto facilitador da construção de equipes eficazes de trabalho. Esse autor enfatiza ser a comunicação o fator que permite a compreensão de outras perspectivas, sejam humanas ou organizacionais, perspectivas essas que permeiam todas as relações de trabalho, notadamente a troca e o fornecimento de feedback.

Em função dos aspectos levantados pelos principais teóricos sobre os temas Cultura Organizacional, Comunicação e orientação para o cliente, o presente estudo baseiase nas seguintes questões de pesquisa:

Q1. Quais são os indicadores capazes de medir traços culturais que dão suporte à orientação para o cliente por parte de uma organização e que devem ser comunicados ao público interno?

Q2. Quais são os indicadores capazes de medir o grau de orientação para o cliente apresentado por uma organização?

\section{Buscando traços culturais voltados ao cliente}

A pesquisa desse estudo é do tipo exploratória (SELLTIZ et al., 1972) e foi realizada junto a uma amostra não-probabilística, com sujeitos selecionados por julgamento e em empresas da Grande São Paulo, envolvendo funcionários de cargos gerenciais em nível equivalente a, no mínimo, coordenador ou supervisor e com escolaridade superior. 
ORIENTAÇÃO PARA O CLIENTE, COMUNICAÇÃO E CULTURA ORGANIZACIONAL •

ROBERTO CODA, ANA CAROLINA MACIEL DA SILVA, MAURO NEVES GARCIA E DIRCEU DA SILVA

O questionário foi desenvolvido com base em pesquisa bibliográfica sobre os construtos desenvolvidos nos campos de Marketing e Cultura Organizacional e em uma pesquisa qualitativa composta de duas atividades principais - a análise de um caso prático e a análise de conteúdo. A análise do caso prático estudou uma organização do setor de telecomunicações em processo de implementação da orientação ao cliente. Já a análise de conteúdo dedicou-se a investigar exemplares especiais de uma publicação brasileira que trazia um diagnóstico cultural completo das organizações premiadas pela excelência em serviços ao cliente. Além disso, também para subsidiar o processo de geração das assertivas para medir valores culturais e orientação ao cliente, foi realizada uma análise sistemática das escalas publicadas nos estudos de Deshpande, Farley, Webster (1993), Homburg e Pflesser (2000), Jaworski e Kohli (1993), Narver e Slater (1990) e Whiteley (1999).

A escala desenvolvida ficou constituída por 60 questões do tipo fechadas, sendo 24 assertivas sobre valores culturais que, a priori, suportam uma orientação ao cliente, e 36 assertivas sobre práticas ou comportamentos organizacionais típicos de uma orientação ao cliente; a escala adotada é do tipo Likert, com sete pontos, variando de $1=$ discordo totalmente até 7 = concordo totalmente. Em termos das dimensões obtidas nessa fase para o construto valores culturais, foi possível identificar oito categoriais, consideradas neste estudo como pré-fatores de investigação: foco na satisfação do cliente; inovação; excelência; flexibilidade; comunicação aberta; interesse pelos funcionários; trabalho em equipe; e confiança. De modo análogo, para o construto práticas orientadas ao cliente, também foram obtidas oito categorias, a saber: compromisso com o cliente; conhecimento do cliente; disseminação das informações do cliente; reação às informações dos clientes; liderança; integração dos departamentos; processos e normas flexíveis; e capacitação e recompensa.

\section{Cultura organizacional e orientação ao cliente: traços obtidos}

A pesquisa de campo gerou respostas de 250 sujeitos, sendo $66 \%$ pertencentes ao sexo masculino, $48,4 \%$ ocupantes do cargo de gerente ou superintendente, $23,2 \%$ pertencentes ao departamento Comercial e $23,2 \%$ ao departamento de Marketing. A minoria trabalha há menos de dois anos na empresa $(22,8 \%), 51,2 \%$ trabalham em empresas com mais de 1.000 funcionários e 65,6\% trabalham em empresas multinacionais.

Os dados multivariados, relativos aos construtos valores culturais e orientação ao cliente, foram analisados por meio da técnica de análise fatorial exploratória. Para o tratamento dos dados, adotaram-se duas etapas distintas. O software SPSS versão 10.0 foi utilizado em ambas as etapas.

Na primeira etapa, foram realizados os testes Kaiser-Meyer-Olkin (KMO) e Esfericidade de Bartlett, tradicionalmente considerados para subsidiar a validade de utilização do 
ORIENTAÇÃO PARA O CLIENTE, COMUNICAÇÃO E CULTURA ORGANIZACIONAL •

ROBERTO CODA, ANA CAROLINA MACIEL DA SILVA, MAURO NEVES GARCIA E DIRCEU DA SILVA

método de análise fatorial (HAIR et al, 1998; SPSS, 1999). Os valores obtidos nos testes Esfericidade de Bartlett (Chi-quadrado $=3858,287 ; \mathrm{p}<0,0001$ para valores culturais e Chi-quadrado $=6075,374 ; \mathrm{p}<0,0001$ para orientação ao cliente $)$ e $\mathrm{KMO}(0,946$ para valores culturais e 0,957 para orientação ao cliente) indicam que os dados poderiam ser efetivamente tratados pelo método de análise fatorial.

No tocante à variância total, foi verificado se os fatores encontrados explicam a maior parte das variáveis, ou seja, mais de $50 \%$ da variância total. Pelos resultados, verificase que quatro fatores explicam $64,142 \%$ da variância total das 24 variáveis que medem valores culturais, e cinco fatores explicam $62,348 \%$ da variância total das 36 variáveis que medem orientação ao cliente.

Para a matriz de componentes principais associada às variáveis de valores culturais, a rotação Varimax forneceu a melhor matriz para a interpretação dos fatores. Já para a matriz associada às variáveis do construto orientação ao cliente, a rotação Equamax forneceu o melhor resultado. O tratamento seguiu as sugestões de Pett, Lackey e Sullivan (2003), segundo as quais variáveis com cargas em diferentes fatores deverão ser agrupadas no fator a que sejam conceitualmente mais relacionadas.

\section{Ajuste do modelo}

O passo final na análise fatorial consiste em determinar o ajuste do modelo. É desejável que as contagens de resíduos maiores que 0,05 ou $5 \%$ estejam em menos que $50 \%$ dos dados. Pelos resultados gerados, verifica-se que o modelo está ajustado para ambos os construtos (valores culturais, resíduos > 5\% estão em $28 \%$ dos dados; orientação ao cliente, resíduos $>5 \%$ estão em $20 \%$ dos dados).

\section{Consistência interna}

Como a intenção é validar o instrumento, deve-se também determinar a confiabilidade interna dos dados obtidos. Para isso, foi utilizado um método de verificação da consistência interna denominado coeficiente alfa de Cronbach, reconhecido como o mais popular e mais usado por pesquisadores da área (YU, 2001).

Segundo Cronbach (1996) e Churchill Jr. (1999), valores entre 0,600 e 0,800 são considerados bons para uma pesquisa exploratória, mostrando que os dados são confiáveis e que o instrumento tem boa qualidade para interpretação. E foi esse o resultado obtido depois de realizados os testes para cada uma das subescalas que compõem os fatores das matrizes que medem valores culturais (Fator 1: 0,9316; Fator 2: 0,8651; Fator 3: 0,8672) e orientação ao cliente (Fator 1: 0,9216; Fator 2: 0,8833; Fator 3: 0,9091; Fator 4: 0,8671). 
ORIENTAÇÃO PARA O CLIENTE, COMUNICAÇÃO E CULTURA ORGANIZACIONAL •

ROBERTO CODA, ANA CAROLINA MACIEL DA SILVA, MAURO NEVES GARCIA E DIRCEU DA SILVA

\section{Identificação e nomeação dos fatores}

A seguir, acham-se apresentados os fatores obtidos para os dois construtos tratados no estudo após a realização dos testes e análises estatísticas pertinentes (tabelas 1 e 2).

Para o construto valores culturais, a composição final abrange 21 indicadores reunidos em três fatores distintos. Para o construto orientação ao cliente, a composição final inclui 32 indicadores reunidos em quatro fatores. Em relação aos pré-fatores uti-

\section{Tabela 1. Formação dos fatores na análise fatorial pelo método Varimax da escala de valores culturais que suportam a orientação ao cliente e respectivas cargas fatoriais}

\begin{tabular}{|c|c|}
\hline Valores culturais que suportam a orientação ao cliente & \multirow{2}{*}{$\begin{array}{r}\text { Carga } \\
\text { fatorial }\end{array}$} \\
\hline Fator 1 = Interesse pelos funcionários & \\
\hline A empresa valoriza funcionários de bom desempenho. & 0,786 \\
\hline A empresa estimula os funcionários a tomarem decisões. & 0,758 \\
\hline Os líderes confiam no bom trabalho das pessoas sem precisar vigiá-las. & 0,721 \\
\hline Os funcionários expressam suas opiniões, idéias e sugestões livremente na empresa. & 0,668 \\
\hline $\begin{array}{l}\text { A empresa enfatiza que as relações criadas com seus funcionários, } \\
\text { clientes e acionistas sejam baseadas na confiança. }\end{array}$ & 0,575 \\
\hline A empresa valoriza as idéias diferentes, não convencionais. & 0,567 \\
\hline A empresa reconhece a contribuição de cada funcionário investindo em sua capacitação. & 0,516 \\
\hline $\begin{array}{l}\text { Na empresa as pessoas são estimuladas a implementar coisas novas, } \\
\text { ainda que cometam alguns erros. }\end{array}$ & 0,554 \\
\hline A empresa promove o diálogo sincero e transparente com seus funcionários. & 0,470 \\
\hline A empresa se preocupa em manter os funcionários informados. & 0,408 \\
\hline A empresa acredita no lema "Cuide de seus funcionários e eles cuidarão de seus clientes". & 0,434 \\
\hline \multicolumn{2}{|l|}{ Fator 2 = Trabalho em equipe } \\
\hline $\begin{array}{l}\text { A empresa promove rápidas mudanças quando detecta que suas normas } \\
\text { e regras dificultam o andamento das atividades. }\end{array}$ & 0,726 \\
\hline A empresa estimula que as atividades dos diferentes departamentos sejam integradas. & 0,719 \\
\hline A empresa incentiva a troca de informações entre departamentos. & 0,707 \\
\hline Na empresa há um forte espírito de colaboração entre as pessoas. & 0,565 \\
\hline Todos acreditam serem responsáveis pelo sucesso da empresa. & 0,527 \\
\hline \multicolumn{2}{|l|}{ Fator 3 = Excelência da satisfação do cliente } \\
\hline A empresa acredita na idéia: o cliente em primeiro lugar! & 0,749 \\
\hline Para realizar bem suas atividades, a empresa acredita ser fundamental ouvir o cliente. & 0,709 \\
\hline A empresa é totalmente comprometida com a idéia de qualidade. & 0,696 \\
\hline A empresa sabe que o cliente é quem define a qualidade de seus produtos e serviços. & 0,667 \\
\hline Melhorar continuamente a forma de trabalhar é um dos lemas da empresa. & 0,566 \\
\hline
\end{tabular}




\section{Tabela 2. Formação dos fatores na análise fatorial pelo método Equamax da escala de práticas orientadas ao cliente e respectivas cargas fatoriais}

\begin{tabular}{|c|c|}
\hline Práticas orientadas ao cliente & \multirow{2}{*}{$\begin{array}{r}\text { Carga } \\
\text { fatorial }\end{array}$} \\
\hline Fator 1 = Respeito pelo cliente & \\
\hline $\begin{array}{l}\text { A empresa demonstra disposição em quebrar regras ocasionalmente e ceder ao cliente } \\
\text { quando a situação requer, de modo a assegurar a sua satisfação. }\end{array}$ & 0,820 \\
\hline $\begin{array}{l}\text { As decisões tomadas na empresa priorizam o atendimento das necessidades } \\
\text { dos clientes, e não o atendimento de politicas e interesses internos. }\end{array}$ & 0,565 \\
\hline $\begin{array}{l}\text { As novas idéias ou sugestões dadas pelos funcionários são usadas nas melhorias } \\
\text { dos produtos e serviços ao cliente. }\end{array}$ & 0,535 \\
\hline $\begin{array}{l}\text { A empresa promove mudanças com rapidez para atender as exigências } \\
\text { ou necessidades dos clientes. }\end{array}$ & 0,527 \\
\hline $\begin{array}{l}\text { As informações dos clientes são usadas no processo de desenvolvimento ou melhoria } \\
\text { de produtos e serviços da empresa. }\end{array}$ & 0,525 \\
\hline $\begin{array}{l}\text { Quando algo importante acontece com o cliente no mercado, a empresa } \\
\text { fica sabendo rapidamente. }\end{array}$ & 0,521 \\
\hline $\begin{array}{l}\text { Quando algum processo interno está prejudicando a satisfação do cliente, } \\
\text { a empresa rapidamente trata de alterá-lo ou até eliminá-lo. }\end{array}$ & 0,473 \\
\hline $\begin{array}{l}\text { A empresa atende as necessidades incomuns de seus clientes porque tem } \\
\text { a capacidade de oferecer-lhes produtos e serviços personalizados. }\end{array}$ & 0,451 \\
\hline $\begin{array}{l}\text { A empresa sabe quais atributos de seus produtos ou serviços são mais valorizados } \\
\text { pelos clientes. }\end{array}$ & 0,445 \\
\hline $\begin{array}{l}\text { Ao serem identificados problemas de qualidade com o cliente, a empresa } \\
\text { age rapidamente para solucioná-los. }\end{array}$ & 0,435 \\
\hline Fator 2 = Qualidade no atendimento ao cliente & \\
\hline $\begin{array}{l}\text { A empresa regularmente faz pesquisa de satisfação dos clientes para avaliar a qualidade } \\
\text { de seus produtos ou serviços. }\end{array}$ & 0,758 \\
\hline $\begin{array}{l}\text { A empresa oferece aos seus funcionários treinamentos voltados a promover } \\
\text { melhorias no atendimento ao cliente. }\end{array}$ & 0,714 \\
\hline $\begin{array}{l}\text { Os funcionários que realizam seus maiores esforços para servirem bem os clientes } \\
\text { são formalmente reconhecidos pela empresa, seja em dinheiro ou não. }\end{array}$ & 0,557 \\
\hline $\begin{array}{l}\text { Os líderes promovem continuamente a filosofia de qualidade voltada para o cliente } \\
\text { por toda a empresa, usando todas as formas possíveis de comunicação para reforçá-la. }\end{array}$ & 0,528 \\
\hline As remunerações estão também atreladas à qualidade do serviço ou à satisfação do cliente. & 0,520 \\
\hline $\begin{array}{l}\text { As reclamações dos clientes são regularmente analisadas de modo a identificar } \\
\text { problemas de qualidade. }\end{array}$ & 0,477 \\
\hline $\begin{array}{l}\text { Grupos de trabalho se reúnem para propor solucões, conceitos, idéias ou processos } \\
\text { de acordo com as especificações e necessidades dos clientes. }\end{array}$ & 0,460 \\
\hline Fator 3 = Conhecimento sobre o cliente & \\
\hline $\begin{array}{l}\text { Os resultados de eventuais pesquisas de satisfação realizadas com o cliente são } \\
\text { comunicados para toda a empresa. }\end{array}$ & 0,425 \\
\hline $\begin{array}{l}\text { As informações sobre mercados e clientes são compartilhadas com todos } \\
\text { os departamentos. }\end{array}$ & 0,736 \\
\hline $\begin{array}{l}\text { A empresa compartilha livremente com seus funcionários informações sobre clientes, } \\
\text { objetivos estratégicos, vendas e resultados. }\end{array}$ & 0,626 \\
\hline
\end{tabular}


ORIENTAÇÃO PARA O CLIENTE, COMUNICAÇÃO E CULTURA ORGANIZACIONAL •

ROBERTO CODA, ANA CAROLINA MACIEL DA SILVA, MAURO NEVES GARCIA E DIRCEU DA SILVA

\begin{tabular}{|l|c|}
\hline $\begin{array}{l}\text { Funcionários de todos os cargos conhecem bem os produtos e serviços que a empresa } \\
\text { oferece aos seus clientes. }\end{array}$ & 0,593 \\
\hline $\begin{array}{l}\text { Regularmente os departamentos da empresa se reúnem para compartilhar } \\
\text { informações sobre os clientes, discutir novas tendências e desenvolvimentos. }\end{array}$ & 0,585 \\
\hline $\begin{array}{l}\text { Em vez de competirem, as diferentes áreas da empresa cooperam entre si para } \\
\text { alcançar as metas comuns de satisfação do cliente. }\end{array}$ & 0,475 \\
\hline $\begin{array}{l}\text { Em todos os níveis, os funcionários têm autonomia para tomarem iniciativas } \\
\text { próprias quando a rápida ação é necessária para atender bem o cliente. }\end{array}$ & 0,473 \\
\hline $\begin{array}{l}\text { Mesmo os departamentos que não têm contato direto com os clientes fazem um bom } \\
\text { trabalho para contribuir com o processo global de atendimento ao cliente. }\end{array}$ & 0,465 \\
\hline $\begin{array}{l}\text { Os líderes sempre envolvem os funcionários no planejamento de ações relativas } \\
\text { à satisfação do cliente. }\end{array}$ & 0,432 \\
\hline $\begin{array}{l}\text { Fator } 4 \text { = Compromisso com o cliente } \\
\text { As promessas feitas aos clientes são sempre cumpridas. }\end{array}$ & 0,716 \\
\hline A empresa não promete aos clientes mais do que ela é capaz de cumprir. & 0,705 \\
\hline Os líderes compreendem com clareza as exigências dos clientes. & 0,548 \\
\hline $\begin{array}{l}\text { No dia-a-dia, percebe-se que toda a empresa está extremamente comprometida } \\
\text { em trabalhar para satisfazer o cliente. }\end{array}$ & 0,505 \\
\hline Os líderes demonstram por suas atitudes que a satisfação do cliente é importante. & 0,494 \\
\hline
\end{tabular}

lizados, observou-se uma aderência aos aspectos teóricos de ambos os construtos, mas, por outro lado, expressiva redução de categorias, agora consideradas fatores. Além disso, o número de indicadores dentro de cada fator obtido aumentou significativamente, dada a inclusão no agrupamento de itens que estavam associados a outras categorias.

\section{Considerações finais}

O estudo buscou determinar a natureza dos inter-relacionamentos entre as variáveis e obter um instrumento final com um número simplificado e otimizado de variáveis. Tal objetivo foi alcançado, uma vez que um expressivo número de indicadores continuou a aparecer nos agrupamentos obtidos e nomeados como fatores específicos. $\mathrm{O}$ estudo resultou em uma redução dos pré-fatores de 16 para sete, sendo três fatores para o construto cultura organizacional e quatro para orientação ao cliente, ratificando os aspectos teóricos levantados sobre Cultura Organizacional e orientação para o cliente. Todavia, não houve uma redução significativa do número inicial de assertivas, uma vez que, das 60 iniciais, apenas sete assertivas foram eliminadas do instrumento final pelas análises fatoriais. Esse fato pode ser explicado pelo motivo de que as assertivas foram criadas com base no trabalho dos principais autores da área, sendo possível concluir, assim, que os pressupostos teóricos definidos por eles se refletem efetivamente nas práticas e políticas empresariais voltadas ao gerenciamento da relação empresa e seus clientes. 
ORIENTAÇÃO PARA O CLIENTE, COMUNICAÇÃO E CULTURA ORGANIZACIONAL •

ROBERTO CODA, ANA CAROLINA MACIEL DA SILVA, MAURO NEVES GARCIA E DIRCEU DA SILVA

Entre as limitações deste trabalho, uma relaciona-se à amostra obtida. Dado que o público-alvo consultado é representado por ocupantes de cargos gerenciais ou superiores a esse nível hierárquico, seria necessário que esse estudo fosse estendido tanto a ocupantes de cargos inferiores como também a integrantes de organizações localizadas em outras regiões brasileiras, a fim de coletar novos indicadores ou então se identificar uma eventual reconfiguração ou redefinição dos atuais.

Uma outra limitação refere-se ao elenco de indicadores utilizados no estudo para medir valores culturais e orientação ao cliente. O conjunto de indicadores e assertivas selecionado não pode ser considerado como único e universal, podendo existir outros que, uma vez incorporados ao instrumento aqui obtido, poderão aprimorá-lo por contemplar outros aspectos que venham a se mostrar também relevantes.

Como se pode constatar junto a empresas de diferentes portes e pertencentes a diferentes setores industriais no Brasil, é grande a preocupação de realizar investimentos em planos e atividades de fidelização de clientes, em campanhas de comunicação com os clientes e mesmo na criação de departamentos dedicados a dar atenção ao cliente, sem, contudo, reconhecer, ou mesmo deixando de lado, a importância para o fato de que a cultura organizacional deve estar alinhada a esse tipo de estratégias empresariais. Sem a existência de crenças e valores compartilhados que apóiem a adoção de uma orientação ao cliente, a implantação desse tipo de filosofia corre o risco de cair no vazio ou, então, não ser devidamente implementada.

Em face desse cenário, o presente estudo oferece uma contribuição metodológica com amplas implicações práticas, uma vez que o instrumento de medição dele derivado permite que as organizações brasileiras, se assim desejarem, identifiquem o respectivo grau atual de orientação ao cliente. Tomando como base esse diagnóstico inicial, em que possíveis oportunidades de melhoria são reveladas, os gerentes podem estabelecer ações para que a organização desenvolva e cultive valores e práticas culturais essenciais à orientação ao cliente.

Além desses aspectos, as contribuições do presente estudo, tais como a investigação teórica e prática dos construtos orientação ao cliente e cultura organizacional, e os indicadores de medição desenvolvidos oferecem uma base para a realização de estudos posteriores que testem as proposições de pesquisa sugeridas por Deshpande $\&$ Webster (1989).

Embora possível, cumpre registrar também que uma análise das prováveis relações existentes entre os construtos valores culturais e orientação ao cliente por meio dos indicadores adotados não foi contemplada no presente estudo, devendo fazer parte de pesquisas voltadas para a ampliação do conhecimento nesse campo da gestão de empresas. 
ORIENTAÇÃO PARA O CLIENTE, COMUNICAÇÃO E CULTURA ORGANIZACIONAL •

ROBERTO CODA, ANA CAROLINA MACIEL DA SILVA, MAURO NEVES GARCIA E DIRCEU DA SILVA

Finalmente, o presente trabalho oferece também a possibilidade de desenvolvimento e teste de um modelo de cultura organizacional orientada ao cliente. Para tanto, considera-se necessário ampliar o escopo do atual instrumento de medição no sentido de incluir como indicadores aspectos que contemplem, além de valores e comportamentos, normas e artefatos culturais que seriam considerados típicos de uma empresa efetivamente voltada para o cliente, já que esses outros elementos são também formadores da cultura de uma organização, conforme apontado pela expressiva maioria dos especialistas nesse tema.

\section{Referências}

AYELET, Baron. Turning strategy into action. Strategic Communication Management, London, v. 8, n. 5, p. 13, Aug./Sept. 2004.

CABRERA, Elizabeth F.; BONACHE, Jaime. An expert HR system for aligning organizational culture and strategy. Human Resources Planning, New York, v. 22, n. 1, p. 51-60, 1999.

CHENEY, George; CHRISTENSEN, Lars Thoger. What should public relations theory do, practically speaking? Journal of Communication Management, Bingley, v. 10, n. 2, p. 100-102, 2006.

CHURCHIL JR., Gilbert A. Marketing research: methodological foundations. 7. ed. New York: Thomson Publishing, 1999.

CRONBACH, Lee J. Fundamentos da testagem psicológica. 5. ed. Porto Alegre: Artes Médicas, 1996.

DAY, George. S. What does it mean to be market-driven? Business Strategy Review, Oxford, v. 9, n. 1, p. 1-14, 1998.

DESHPANDE, Rohit; FARLEY, John U.; WEBSTER, Frederick. Corporate culture, customer orientation and innovativeness in japanese firms: a quadrad analysis, Journal of Marketing, Chicago, v. 57, n.1, p. 23-27, 1993.

Organizational culture and marketing: defining the research agenda. Journal of Marketing, Chicago, v. 53, n. 1, p. 3-15, 1989.

DESSLER, Gary. Management: leading people and organizations in the $21^{\text {st }}$ century. New Jersey: Prentice Hall, 1998.

DRUCKER, Peter F. The practice of management. New York: Harper and Row Publishers, 1954.

EDELMAN, Andrew J. Does your company culture drive away your best workers? How to build trust, cooperation \& teamwork. Supervision, Burlington, v. 67, n. 12, p. 12-14, 2006.

HAIR, Joseph F. et al. Multivariate data analysis. 5. ed. New Jersey: Prentice Hall, 1998.

HOFSTEDE, Geert. Cultures and organizations: software of the mind. 2. ed. New York: McGraw Hill, 1991.

. et al. Measuring organizational cultures: a qualitative and quantitative study across twenty cases. Administrative Science Quarterly, Ithaca, v. 35, n. 2, p. 286-317, 1990. 
ORIENTAÇÃO PARA O CLIENTE, COMUNICAÇÃO E CULTURA ORGANIZACIONAL •

ROBERTO CODA, ANA CAROLINA MACIEL DA SILVA, MAURO NEVES GARCIA E DIRCEU DA SILVA

HOMBURG, Christian; PFLESSER, Christian A. Multiple-layer model of market-oriented organizational culture: measurement issues and performance. Journal of Marketing Research, Chicago, v. 37, n. 4, p. 449-462, 2000.

JAWORSKI, Bernard J.; KOHLI, Ajay K. Market orientation: antecedents and consequences. Journal of Marketing, Chicago, v. 57, n. 3, p. 53-70, 1993.

KOHLI, Ajay K.; JAWORSKI, Bernard J. Market orientation: the construct, research propositions and managerial implications. Journal of Marketing, Chicago, v. 54, n. 2, p. 1-19, 1990.

KOTLER, Phillip. Marketing para o século XXI. 5. ed. São Paulo: Futura, 2000.

LANDALE, Anthony. Must have EQ. British Journal of Administrative Management, London, n. 57, p. 24-25, Feb./Mar.2007.

MOYNIHAN, Donald; PANDEY, Sanjay. Creating desirable organizational characteristics. Public Management Review, Oxfordshire, v. 8, n. 1, p. 119-140, 2006.

NARVER, John C.; SLATER, Stanley. Additional thoughts on the measurement of market orientation: a comment on Deshpande and Farley. Journal of Market Focused Management, v. 2, n.3, p. 233-236, 1998.

$\mathrm{NASH}$, Jill. A comprehensive campaign helps GAP employees embrace cultural change. Communication World, San Francisco, v. 22, n. 6, p. 42-12, 2005.

NARVER, John C.; SLATER, Stanley. The effect of market orientation on business profitability, Journal of Marketing, Chicago, v. 54, n. 4, p. 20-36, 1990.

OVERHOLT, Miles H.; GRANELL, E. Highlights of the human resource planning society 2002 state-of-the-art \& practice study: managing strategic and cultural and HRM alignment to maximize customer satisfaction and retention. Human Resource Planning, New York, v. 25, n. 2; p. 45-55, 2002.

PETT, Marjorie A.; LACKEY, Nancy R.; SULLIVAN, John J. Making sense of factor analysis: the use of factor analysis for instrument development in health care research. London: Sage Publications, 2003.

PETTIGREW, Andrew M. On studying organizational cultures. Administrative Science Quartely, Ithaca, v. 24, n. 4, p. 570-581, Dec. 1979.

PORTER, Michael E. Estratégia competitiva. 6. ed. Rio de Janeiro: Campus, 1991.

POTTER, Lester R. The communicator as gardener. Communication World, San Francisco, v. 20, n. 2, p. 14-18, 2003.

PRICE, Andrew D. F.; CHAHAL, Kusminder. A strategic framework for change management. Construction Management and Economics, Oxfordshire, v. 24, n. 3, p. 237-251, Mar. 2006.

RAVASI, Davide; SCHULTZ, Majken. Responding to organizational identity threats: exploring the role of organizational culture. Academy of Management Journal, Briar Cliff Manor, v. 49, n. 3, p. 433-458, 2006.

ROBBINS, Stephen P. Comportamento organizacional. 8. ed. São Paulo: LTC Livros Técnicos e Científicos, 1999.

ROUSSEAU, Denise M. Assessing organizational culture: the case for multiple methods. In: SCHNEIDER, Benjamin (org.) Organizational climate and culture. San Francisco: Jossey-Bass, 1990. p. 153-192. 
ORIENTAÇÃO PARA O CLIENTE, COMUNICAÇÃO E CULTURA ORGANIZACIONAL •

ROBERTO CODA, ANA CAROLINA MACIEL DA SILVA, MAURO NEVES GARCIA E DIRCEU DA SILVA

SALLIE-DOSUNMU, Meloney. Communication: a golden ticket? Strategic Communication Management, London, v. 5 , n. 1, p. $5,2001$.

SCHEIN, Edgard. Organizational culture and leadership. 2. ed. San Francisco: Jossey-Bass, 1997.

SCHNEIDER, Benjamin. Organizational climate and culture. San Francisco: Jossey-Bass, 1990.

SELLTIZ, Claire et al. Métodos de pesquisa nas relações sociais. São Paulo: EPU: Edusp, 1972.

SLATER, Stanley; NAVER, John C. Market orientation, customer value and superior performance. Business Horizons, Oxford, v. 37, n. 2, p. 22-29, 1994.

SPSS - STATISTICAL PACKAGE FOR THE SOCIAL SCIENCES. Base 10.0 User's Guide. Chicago: SPSS, 1999.

THOMPSON, Karl; LUTHANS, Francis. Organizational culture: a behavior perspective. In: WEBSTER, Frederick E. Market-driven management: using the new marketing concept to create a customer-oriented company. New York: John Wiley \& Sons, 1994.

WATERHOUSE, Jennifer; LEWIS, Dianne. Communicating culture change. Public Management Review, Oxfordshire, v. 6, n. 3, p. 353-376, 2004.

WEBSTER, Frederick. E., Market-driven management: using the new concept to create a customer-oriented company. New York: John Wiley \& Sons, 1994a.

Defining the new marketing concept. Marketing Management, Chicago, v. 2, n. 4, p. 22-32, 1994b.

WHITELEY, Richard. A empresa totalmente voltada para o cliente. Rio de Janeiro: Campus, 1999.

$\mathrm{YU}$, Chang $\mathrm{H}$. An introduction to computing and interpreting Cronbach coefficient alpha in SAS. In: SAS USER GROUP INTERNATIONAL CONFERENCE, 26. 2001, Long Beach. Proceedings. Disponível em: <seamonkey.ed.asu.edu/ alex/pub/cronbach.doc>. Acesso em: 23 jun. 2001.

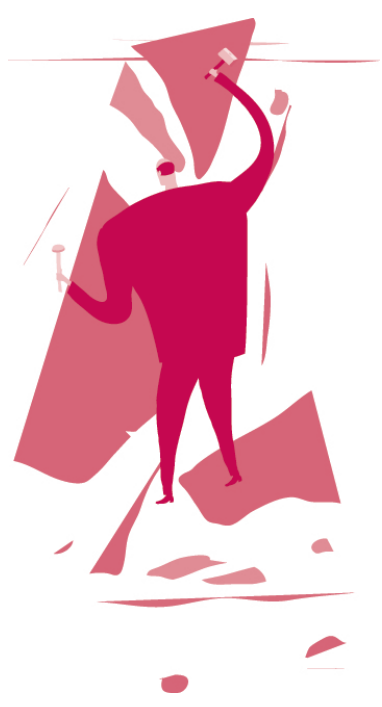

ANO $4 \cdot$ NÚMERO $6 \cdot 1^{\circ}$ SEMESTRE DE $2007 \cdot$ organicom • 47 